\title{
Efflux Pump Inhibitors Derived From Natural Sources as Novel Antibacterial Agents Against Pseudomonas aeruginosa: A Review
}

\author{
Davood Iman Islamieh', Davoud Afshar ${ }^{2}$, Masoud Yousefi ${ }^{3}$, Davoud Esmaeili ${ }^{*}$ \\ ${ }^{1}$ Department of Microbiology and Applied Microbiology Research Center, Systems Biology and Poisonings Institute, Baqiyatallah \\ University of Medical Sciences, Tehran, Iran \\ ${ }^{2}$ Department of Microbiology, School of Medicine, Zanjan University of Medical Sciences, Zanjan, Iran \\ ${ }^{3}$ Department of Microbiology, Faculty of Medicine, Birjand University of Medical Sciences, Birjand, Iran
}

Corresponding Author: Davoud Esmaeili, PhD, Associate Professor, Department of Microbiology and Applied Microbiology Research Center, Systems Biology and Poisonings Institute, Baqiyatallah University of Medical Sciences, Tehran, Iran. Tel/Fax: +9821-82483422, Email: esm114@gmail.com

Received May 17, 2018; Accepted July 4, 2018; Online Published September 30, 2018

\begin{abstract}
Pseudomonas aeruginosa is one of the most important opportunistic human pathogen that can lead to serious nosocomial infections. The treatment of pseudomonas infections due to the high emergence of resistance to antimicrobial agents is challenging. One of the mechanisms of antibiotic resistance in $P$. aeruginosa is the active efflux pumps. To date, 11 different efflux pumps of the RND family in P. aeruginosa that enable the efflux of antibiotics/anti-microbial production have been detected. These pumps include MexAB-OprM, MexCD-Opr], MexEF-OprN, MexGHI-OpmD, MexJK-OprM/OpmH, MexMN, MexPQ-OpmE, MexVW-OprM, MexXY-OprM, TriABCOpmH, and MuxABC-OpmB. Recently, Efflux Pump Inhibitors (EPIs) have been considered as novel antimicrobial agents to overcome drug efflux mechanisms of bacterial pathogens. In recent years, numerous EPIs have been identified or synthesized for multi-drug resistant (MDR) bacteria; these include existing pharmacologic drugs, naturally occurring compounds, and synthetic derivatives thereof. This review describes the current progress in the assessment and development of EPI for using against $P$. aeruginosa.

Keywords: Pseudomonas aeruginosa, Efflux System, Antibiotic Resistance, Antibacterial Agent, Efflux Pump Inhibitor

Citation: Iman Islamieh D, Afshar D, Esmaeili D. Efflux pump inhibitors derived from natural sources as novel antibacterial agents against Pseudomonas aeruginosa: a review. Int J Med Rev. 2018;5(3):94-105. doi:10.29252/IJMR-050303.
\end{abstract}

\section{Introduction}

Pseudomonas aeruginosa is a gram-negative bacterium that can survive in different environments, including soil, plants, and animals. Moreover, it is the most opportunistic human pathogen, particularly in immunocompromised patients, and has been recognized as one of the five main pathogens in nosocomial infections. ${ }^{1}$ Pseudomonas diseases are mainly seen in urinary tract infections (UTIs), burn wounds, and pulmonary infections such as cystic fibrosis (CF). Mortality rates in $P$. aeruginosa infections are up to $50 \%$ and $70 \%$ in patients with bacteremia and nosocomial pneumonia, respectively. ${ }^{2} P$. aeruginosa infections are diverse, because they have a multiplicity of resistance mechanisms and have developed different metabolic and nutritional pathways. ${ }^{3}$

Of the resistance mechanisms to be noted is the formation of biofilms, which provide better protection against different antibiotics and body defense systems. ${ }^{3}$ Additionally, low outer cell membrane permeability causes intrinsic resistance to antibiotics and increases the number of multi-drug resistance (MDR) strains in $P$. aeruginosa (resistance $\geq 3$ antibiotic classes) ${ }^{4-7}$ Moreover, the high expression of efflux pumps can rapidly expand antibiotic resistance as well. ${ }^{8}$ This review discusses types of efflux pumps, the efflux pumps role in pathogenicity, the importance of efflux pump inhibitors (EPIs), diagnostic methods for their detection, and compounds derived from natural sources that reduce infections caused by MDR $P$. aeruginosa.

\section{Efflux Pumps and Their Importance in Antibiotic Resistance \\ Since the discovery of bacterial efflux pumps in the 1980s, many of them have been characterized. ${ }^{9}$ Efflux pumps are membrane proteins and participate in the extrusion of antibiotics and chemicals such as organic solvents, dyes, detergents, intermediate molecules in cellular communications, metabolic products, and biocides in prokaryote and eukaryote cells. ${ }^{10}$}

Substrates of note of the efflux pumps are oxazolidinones

Copyright (C) 2018 The Author(s). This is an open-access article distributed under the terms of the Creative Commons Attribution License (http:// creativecommons.org/licenses/by/4.0), which permits unrestricted use, distribution, and reproduction in any medium, provided the original work is properly cited. 
and tetracyclines. In the case of oxazolidinones, since the potency against gram-positive pathogens fails by intrinsic efflux, they are only used in infections generated by grampositive bacteria. Great efforts are currently being made toward producing oxazolidinone derivatives that confuse the relevant gram-negative efflux pump. ${ }^{11}$

In the tetracycline class, there are newer compounds that differ from their progenitors by having lower affinity for efflux pumps and the so-called glycylcycline subclass. For example, the new glycylcycline tigecycline confuses a number of tetracycline-specific efflux pumps of gram-negative bacteria. ${ }^{12}$ Recently it has been shown that some EPIs can increase the activity of tigecycline. ${ }^{11}$ Of course, the majority of efflux systems are not drug-specific proteins and extrude a broad spectrum of compounds from bacteria. ${ }^{13}$

Although efflux pumps are very effective in the growth of multidrug-resistant bacteria, it is obvious that the efflux of drugs is not their main function. These pumps contribute to various processes of bacterial pathogenesis, such as participation in the escape from host defense mechanisms, colonization, biofilm production, and toxin production. ${ }^{14}$ Therefore, by understanding the mechanism of action pumps and ways to dominate their pathogenicity, we can establish a promising path for novel antibiotics. ${ }^{15,16}$

Efflux pumps were reported both in gram-negative and gram-positive bacteria and even in a few eukaryotic cells. ${ }^{17}$ In gram-negative bacteria, the majority of the efflux pumps have a three-part structure that overpasses both inner and outer membranes. This assembly directly extrudes substrates from the intracellular to the extracellular and causes the ineffectiveness of drugs. ${ }^{18}$

The bacterial multidrug efflux pumps are categorized into the RND (resistant nodulation division superfamily), the MFS (major facilitator superfamily), the SMR (small multidrug resistance family of drug metabolite transporters [DMT] superfamily e.g., E. coli EmrE), the ATP-binding cassette (ABC) superfamily, and the MATE (multidrug and toxic efflux family). ${ }^{16,19}$ For the active transport of substrates, those gain energy from proton motive force (RND, MFS, and SMR), $\mathrm{Na}+$ dependent (MATE), or by ATP hydrolysis (ABC).,216,20 RND and MFS are the most frequently used systems. RND is in gram-negative bacteria only (represented by $P$. aeruginosa Mex pumps and E. coli AcrAB-TolC), but MFS is in both gram-negative and gram-positive bacteria. ${ }^{21}$

\section{Efflux Pumps in Pseudomonas aeruginosa}

$P$. aeruginosa has various efflux pumps such as RND and MFS superfamilies; however, the main efflux pump belongs to the RND family. ${ }^{22,23}$ Table 1 shows the major transporters identified in $P$. aeruginosa plus the major antibiotic classes they pass. The RND pumps, which exchange antibiotic/ proton, are located in the inner membrane (IM). They must interact with the outer membrane $(\mathrm{OM})$ channel through periplasmic linker protein (also known as membrane fusion protein) and, therefore, produces a tripartite complex. OM ensures that the removed substrate does not stay in the periplasm (Figure 1). ${ }^{24,25}$

There are 11 types of RND efflux pumps in $P$. aeruginosa that differ in their substrates and release of multi-class drugs ${ }^{26}$ Of these, MexXY-OprM and MexAB-OprM are more important due to their high prevalence in clinical strains and their potency in expelling various classes of antibiotics. ${ }^{27}$ These 2 systems are permanently expressed with an amount specified in the wildtype strains and are responsible for the intrinsic resistance to fluoroquinolones. ${ }^{1,26}$ Of course, the expression of MexAB-OprM is greater than that of MexXY-OprM. Both pumps are inducible when expose bacteria with antibiotics. The other pumps (MexEF-OprN, MexJK, MexCD-OprJ, MexGHI-OpmD, MexPQ-OpmE, MexVW-OprM, MexMNOprM, and TriABC) are not in the wild type strains, but are expressed in resistant isolates and may participate in biocide or antibiotic resistance..$^{28}$

The most studied tripartite efflux pumps are the MexABOprM and AcrAB-TolC transporters from P. aeruginosa and

Table 1. Main Efflux Systems in Pseudomonas aeruginosa and Their Antibiotic Substrates

\begin{tabular}{|c|c|c|c|}
\hline Antibiotics & Efflux pump & Pump Family & References \\
\hline Chloramphenicol & $\mathrm{CmlA}$ & MFS & 16,23 \\
\hline Chloramphenicol & MexEF-OprN, MexCD-Opr], MexAB-OprM & RND & $16,31-33$ \\
\hline Erythromycin, Roxythromycin & MexCD-OprJ & RND & 31,33 \\
\hline Macrolides, Lincosamides, Ketolides & MexCD-OprJ, MexAB-OprM, MexXY-OprM & RND & $16,31,32$ \\
\hline Glycylcyclines & MexXY-OprM, MexAB-OprM, MexCD-OprJ & RND & 32,34 \\
\hline$\beta$-lactams & MexAB-OprM, MexCD-OprJ & RND & 32 \\
\hline Aminoglycosides & EmrE homologs & SMR & $23,25,32$ \\
\hline Aminoglycosides & MexXY-OprM, MexAB-OprM & RND & 23,32 \\
\hline Oxazolidinones & MexAB-OprM, MexXY-OprM & RND & $16,31,32$ \\
\hline Fluoroquinolones & MexAB-OprM, MexCD-OprJ, MexEF-OprN, MexXY-OprM, MexVW-OprM & RND & $16,31-33$ \\
\hline Fluoroquinolones & PmpM & MATE & 35 \\
\hline Nalidixic acid, norfloxacin & Orf12-Orf11-Orf10 (plasmid) & $\mathrm{ABC}$ & 31 \\
\hline Trimethoprim & MexCD-OprJ, MexEF-OprN, MexAB-OprM & RND & 36 \\
\hline Tetracyclines & MexCD-Oprj, MexAB-OprM, MexXY-OprM & RND & 20,36 \\
\hline Tetracyclines & Tet A, C, E & MFS & 23 \\
\hline Sulfamides & MexAB-OprM & RND & 27 \\
\hline
\end{tabular}




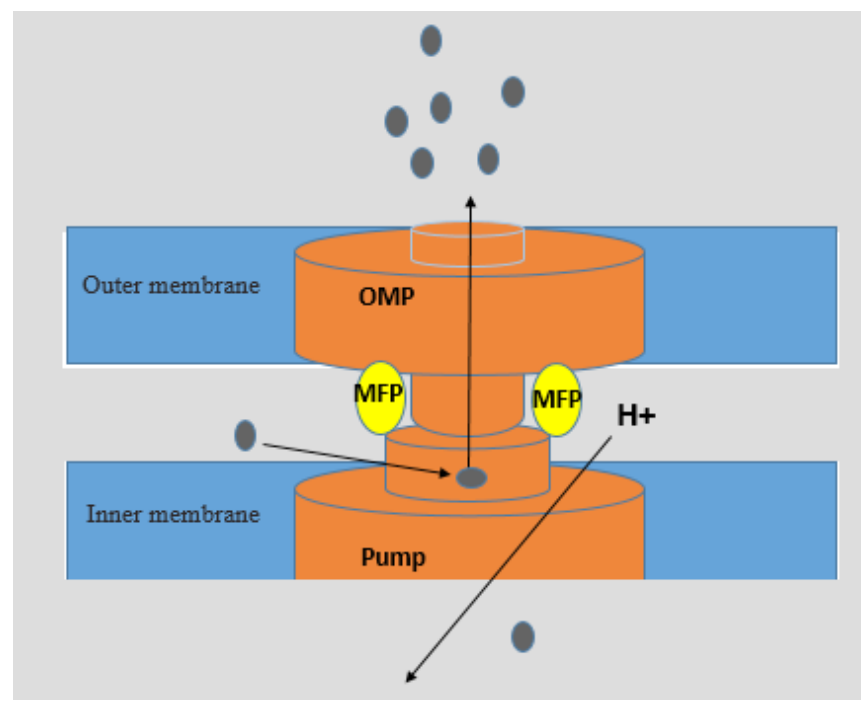

Figure 1. Schematic Representation of Drug Efflux Pumps and Pathway of Drug Efflux Across the IM and OM in Pseudomonas aeruginosa. Efflux transporters in $P$. aeruginosa are three components (a drug-proton transporter in the inner membrane, a membrane fusion protein (MFP) in the periplasmic space, and an outer membrane protein (OMP). For example, in the MexABOprM pump, the IMP is MexB, which identifies specifically with substrates and catalyzes proton dependent drug transport. MFP is MexA and links the OMP and the IMP. These efflux pumps capture the antibiotic from the IM and the periplasm and directly extrude it out of the cells. Finally, OprM plays the role of OMP.

Escherichia coli, respectively. ${ }^{29}$ The inner membrane proteins (IMPs), MexB, and AcrB are similar too, and in research, MexB can be used as a substitute for AcrB. ${ }^{30}$ MexEF-OprN confers resistance to doripenem and meropenem, but this resistance may be caused by the low expression of OrpD porin. ${ }^{27}$

Aminoglycosides have been introduced as poor substrates for efflux pumps due to their very hydrophilic trait. Not long ago, they were proven to be transported by a large number of efflux pumps from the RND superfamily, such as the MexXYOprM pump of $P$. aeruginosa (Table 1 ).

Colistin, the last weapon in the fight against $\operatorname{MDR} P$. aeruginosa, is not effluxed by these pumps. Ciprofloxacin (Cip) and levofloxacin (Lev) are antibiotics that can be substrates for all main RND pumps (Table 1). ${ }^{27}$

\section{EPIs as Novel Therapeutic Instruments}

The continuous prevalence of MDR strains of $P$ aeruginosa have made treatment difficult and necessitated the detection of new antibiotics. According to the decrease in antibiotic expansion, however, it is necessary to search and characterize the compounds that return the activity of older antibiotics against bacteria. ${ }^{37}$ Among these compounds, resistancemodifying agents, more especially EPIs, can be noted. ${ }^{9,38}$ EPIs are the molecules which disorder the process of extruding antibiotics and toxic substances from the bacterial cells with different mechanisms. ${ }^{39}$ Applying EPIs is the most promising approach to inhibiting the multidrug efflux pumps. Indeed, based on their properties and by diverse mechanisms, EPIs can disrupt the function, assembly, and expression of efflux pumps as shown in Figure 2 and Table 2. Moreover, pump inhibitors are applied as diagnostic tools. MC- 207,110 is usually applied for the detection of active efflux pumps in gram-negative pathogen profiling and reserpine for grampositive bacteria. ${ }^{23}$ They can be used as diagnostic tools for the detection of active efflux in pathogens as a mechanism of resistance. For this application, narrow-spectrum inhibitors which allow the gross identification of the transporters that are expressed are preferred.

Compounds such as dinitrophenol (DNP), valinomycin, and carbonylcyanide $m$-chlorophenylhydrazone (CCCP) impacts the energy measure of the cell membrane and suppresses entirely the efflux of various molecules. ${ }^{11}$ Valinomycin collapses the electrochemical gradient generated by potassium ions, whereas DNP and CCCP cause a waste of the proton gradient and collapse the proton-motive force of the cell membrane. ${ }^{40}$ Changing the chemical structure of the drug might attenuate potency against its cellular target. For this reason, no novel antibiotic has been designed to date for gram-negative bacteria. $^{40}$

\section{Multidrug Resistance in Human Cells}

MDR is a critical problem in the treatment of human diseases. One main mechanism that can cause the improvement of $\mathrm{MDR}$ in human cells is drug extrusion by $\mathrm{ABC}$ transporters. $\mathrm{ABC}$ transporters have 2 transmembrane domains and 2 ATP-binding cassettes. ABC transporters present a significant mechanism for supporting CNS tasks. They have diverse substrates, such as drugs and poisons. The cytotoxic drugs applied for MDR in cancer cells are as follows: Vinca alkaloids, microtubule-stabilizing taxanes (docetaxel and paclitaxel), anthracyclines, epipodophyllotoxins, antimetabolites, topotecan, and actinomycin-D. ${ }^{41}$

The family of ABC pumps has been classified into seven subfamilies. Most investigations into members of this family have been conducted with permeability glycoprotein 1 (abbreviated as P-gp), breast cancer resistance protein (BCRP), and multidrug resistance-associated proteins-2 (MRP2). P-glycoprotein 1, also known as multidrug resistance protein 1 (MDR1), creates resistance to antibiotics and cytotoxic drugs. ${ }^{11}$ MDR1 crosses a broad spectrum of substrates which,

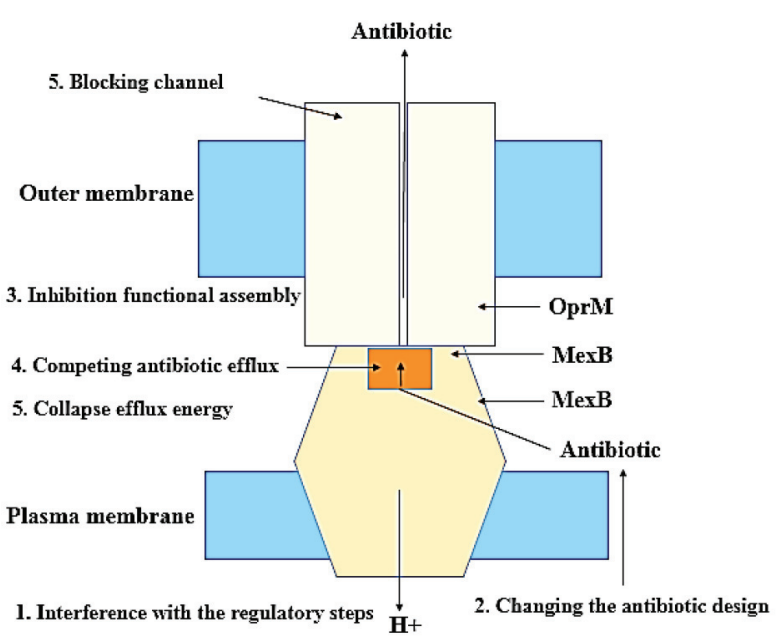

Figure 2. Schematic Showing of an Efflux Pump (MexAB-OprM as an example). The general strategies for efflux pump suppression and the targets that may be influential are displayed. 
Table 2. Mechanisms to Inhibit Drug Efflux, Effective Compounds, and Their Study Methods

\begin{tabular}{|c|c|c|c|}
\hline Mechanism of Action & Example of Inhibitors & Analysis Method & References \\
\hline $\begin{array}{l}\text { Interference in the regulatory steps and suppression of } \\
\text { pump expression }\end{array}$ & Satureja khuzestanica & Real time PCR, RT-PCR & $49-51$ \\
\hline $\begin{array}{l}\text { Alterations in the structure antibiotic so the drug easily } \\
\text { enters the cell, but is not identified by a pump }\end{array}$ & $\begin{array}{l}\text { A chemical modification, e.g., } \\
\text { adds succinate group at taxol }\end{array}$ & Rhodamine 123 accumulations Assay & 40,46 \\
\hline Disruption of pump assembly & $\begin{array}{l}\text { DARPin inhibitors (Ankyrin repeat } \\
\text { proteins) }\end{array}$ & $\begin{array}{l}\text { The interaction between the components of the } \\
\text { efflux pump can be evaluated with the surface } \\
\text { plasmon resonance (SPR) method. }\end{array}$ & 30,52 \\
\hline $\begin{array}{l}\text { Competing inhibition of IMP directly with a high } \\
\text { affinity antibiotic }\end{array}$ & $\begin{array}{l}\text { DARPin inhibitors (Ankyrin repeat } \\
\text { proteins) }\end{array}$ & $\begin{array}{l}\text { Direct interaction between the EPI and the IMP could } \\
\text { be measured by SPR or isothermal calorimetry (ITC) }\end{array}$ & 52 \\
\hline $\begin{array}{l}\text { Connect unmediated to the outer pores and block } \\
\text { them (block exit duct) }\end{array}$ & A number of indole derivatives & $\begin{array}{l}\text { Blocking of the OMP through the tripartite pump } \\
\text { could be determined by suppressing antibiotic efflux. }\end{array}$ & 29 \\
\hline $\begin{array}{l}\text { Disrupt PMF and activity of the efflux pump by } \\
\text { disorder in the proton gradient }\end{array}$ & $\begin{array}{l}\text { Compounds that disrupt the } \\
\text { proton gradient }\end{array}$ & $\begin{array}{l}\text { The measure of the pmf and changes caused by the } \\
\text { effect of EPIs on these could be evaluated by the use } \\
\text { of fluorescent specific compounds for the } \Delta \mathrm{pH} \text { or } \Delta \psi \\
\text { components of the pmf. }\end{array}$ & 53 \\
\hline
\end{tabular}

in addition to chemotherapy, may be used for allergy relief, hypertension, immunosuppression, infections, inflammation, and neurology. ${ }^{42}$

Gemifloxacin is a newer fluoroquinolone and causes a wide range of activity against gram-negative bacteria. ${ }^{43}$ The intracellular accumulation of (14C) erythromycin was performed singly and in the presence of gemifloxacin plus the specific inhibitors of MRP2 and P-gp, the MK571and quinidine, respectively. In addition, gemifloxacin can stimulate the expression of both pumps by activating nuclear hormone receptors such as PXR. Nuclear factors, a superfamily of transcription factors, are stimulated by an excess of endogenous activators such as retinoids, steroids, oxysterols, and bile acids. These activated receptors are then linked to the promoter of the intended gene. ${ }^{44,45}$

Most inhibitor agents with reversal effects can block MDR1 competitively or noncompetitively. Competitive modulators such as verapamil act as a substrate with the cytotoxic drug for cross by the transporter. A noncompetitive inhibitor such as cyclosporin $\mathrm{A}$ is not a substrate but binds to the transporter. This causes a structural change in the pump and, as a result, prevents ATP hydrolysis and the extracellular transfer of the drug. Other noncompetitive inhibitors can be connected to drugs such as paclitaxel (taxol) and can cause chemical change in them. Therefore, P-glycoprotein cannot identify taxol and easily transit the blood brain barrier and approach its target without being extruded by P-glycoprotein. ${ }^{29,46}$

Most natural products extracted from medicinal plants (called secondary metabolites) exhibit anticancer properties. The main classes of secondary metabolites with anticancer activities are phenolics (e.g., flavonoids), terpenoids, and alkaloids. The first natural compound applied as an anticancer substrate was Podophyllotoxin that was extracted from Podophyllum peltatum. Afterward, taxol, vinca alkaloids (vinblastine and vincristine), and chemical derivatives (teniposide and etoposide) were identified as active parts of Taxus brevifolia. ${ }^{47}$

Some secondary metabolites with inhibitory $A B C$ transporters overcome multidrug resistance in cancer cells. For example, the MDR modulatory activity of Chelidonium majus can be helpful in cancer treatment. Furthermore, some flavonoids have been proven to block MDR1-mediated transport mechanisms by directly connecting to the adjacent steroid- and ATP -binding sites. ${ }^{48}$

Therefore, the use of EPIs is effective and can $(a)$ increase the intracellular antibiotic measure, $(b)$ decrease the antibiotic minimal inhibitory concentration (MIC) for the antibiotic, (c) improve the activity of an antibiotic against resistant strains and eradicate the resistant bacteria, and $(d)$ repress the appearance of MDR strains. ${ }^{1,9}$ Nevertheless, for this issue to happen, the physiological mechanisms and structures of efflux pumps must be known. ${ }^{54}$

\section{Types of Efflux Pump Inhibitors}

Entrance of amphipathic compounds is difficult in gramnegative bacteria because of the presence of lipophilicity and an additional outer membrane. ${ }^{55}$ As a result, very few are special for gram-negative bacteria. ${ }^{9} \mathrm{~A}$ lot of synthetic or natural compounds with different natures, such as analogs to an antibiotic, peptidomimetic inhibitors, and other chemical substrates, were examined for their efflux pump inhibition properties against $P$. aeruginosa. ${ }^{40}$

\section{Synthetic Compounds against Pseudomonas aeruginosa}

Synthetic compounds remain one of the major EPIs. Most compounds used as EPI for $P$. aeruginosa overexpressing MexAB-OprM pumps are groups of peptidomimetic molecules with phenylalanine arginine beta naphthylamide $(\mathrm{PA} \beta \mathrm{N})$ as a leading compound. These compounds are known as C-capped dipeptides. $\mathrm{Pa} \beta \mathrm{N}$, or MC-207,110, was the first EPI to be recognized in resistant $P$. aeruginosa and is effective against RND pumps. Therefore, it has been developed for clinical use as an adjuvant (Figure 3a). These inhibitors act with a competitive inhibition approach and are recognized instead of the target antibiotics (quinolones, mainly Lev and Cip) by MexAB-OprM. Until the pumps extrude these inhibitors outside the cell, the antibiotic stays and increases intracellular concentration. ${ }^{54}$ It was also revealed that PA $\beta \mathrm{N}$ can increase the potency of other antibiotics such as macrolides and chloramphenicol; therefore, it is considered as a broad spectrum EPI. ${ }^{56}$ Unfortunately, it expands the penetrance of the OM for other cells which lack the MexAB-OprM pump. 


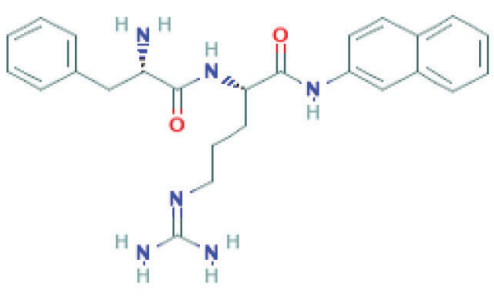

B

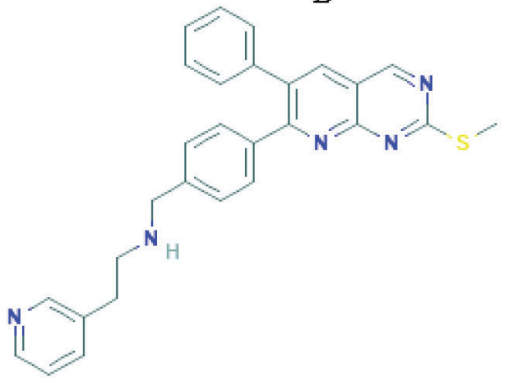

Figure 3. Synthetic EPIs Acting Against P. aeruginosa (a) PA $\beta N$ and its derivatives (MC-02,595, MC-04,124) and (b) pyridopyrimidine derivatives (D13-9001, MBX2319)

This character of PA $\beta \mathrm{N}$ is because of its dicationicity. ${ }^{57}$ Albeit the efficacy of $\mathrm{PA} \beta \mathrm{N}$ on membrane integrity is lower than that of polymyxin $\mathrm{B}$.

In the following, improved the activity and pharmaceutical properties of PA $\beta \mathrm{N}$ and finally discovered MC-04,124 and MC-02,595 (Figure 3A). All these compounds increase the potency of Lev.MC-04,124 (Figure 3A) and show lower toxicity levels, better solubility, more stability in biological fluids, and more activity against $P$. aeruginosa overexpressing efflux pumps. ${ }^{54} \mathrm{MC}-02,595$ exhibited lower toxicity and more stability than the other 2 compounds. ${ }^{58}$

In addition to PA $\beta \mathrm{N}$ derivatives, pyridopyrimidines have also been shown to have high efficiency in inhibiting $P$. aeruginosa efflux pumps (Figure 3B). ${ }^{40}$ These compounds showed higher solubility and improved activity through the import of chloramphenicol (Cam) and fluoroquinolone antibiotics as well as inhibition of the $\beta$-lactam efflux. ${ }^{1,54} \mathrm{D} 13-$ 9001, one of these synthesized compounds, is an analog of ammonium acetic acid; it improves the potency of the Lev and aztreonam and achieved a good safety level in the acute toxicity assay. Hence, it is used as MexAB-OprM EPIs in $P$. aeruginosa. ${ }^{11}$ Finally, another pyranopyridine, MBX2319 (Figure 3B), has recently been characterized as having high activity against Enterobacteriaceae and low activity vs. $P$. aeruginosa. This inhibitor is in the initial phases of optimization and has not been evaluated in in vivo models. ${ }^{57}$

Unfortunately, other than EPI properties, synthetic compounds also permeate up the outer membrane. A number of these inhibitors were confirmed using in vivo models; however, they were prohibited because of toxicity. ${ }^{29,59-61}$ For example, the moieties of PA $\beta \mathrm{N}$ that were applied for activity in $P$. aeruginosa infections caused nephrotoxicity. ${ }^{57}$

Other EPIs that have been studied in detail are quinolines. These compounds inhibit the AcrAB pump and elevate the activity of multiple antibiotics such as cycline, quinolone, and phenicol against gram-negative bacteria. Due to the link of the alkyl side-chain and chlorine to the heterocyclic moiety of quinoline, are synthesized quinolone analogs such as alkoxy-, thioalkoxy-, alkylamino-, and chloro-quinolines that have a slight efficiency against $P$. aeruginosa. ${ }^{11,62}$

On the other hand, quinazolines have also been applied in different areas as EPIs. By adding different active groups to the quinazoline, a variety of quinazoline derivatives with various biological properties have been synthesized. ${ }^{63}$ Among the alkylaminoquinazoline derivatives, the compounds that encompass a morpholine group along with a propyl chain are more effective than the others. ${ }^{64}$

Table 3 shows the compounds that act as EPIs against $P$. aeruginosa. The purpose of the term EPI here is that some of the compounds were identified according to their synergism with antibiotics, whereas no analysis was performed to study non-specific impacts (e.g., membrane permeabilization) or survey the mechanism of inhibition. ${ }^{29}$

\section{Natural Compounds Against Pseudomonas aeruginosa}

The use of natural knowledge of traditional physicians provides helpful data for investigating new drugs. ${ }^{71}$ The potential antibacterial characteristics of diverse natural medicinal substances are being widely studied in different parts of the world. ${ }^{72}$ Among these natural substances, it has been established that many medicinal plant substances have activity against $P$. aeruginosa. ${ }^{55,73,74}$ Plants are extracted with water, methanol, and chloroform. The important issue is that none of the solvents should have antimicrobial activity at the applied concentration. ${ }^{37}$ Plant antimicrobials have not been applied in systemic infections. ${ }^{55}$ Additionally, some plant antimicrobial peptides demonstrate side effects against mammalian cells. Therefore, before being prescribed in new clinical treatments, their toxicity to humans must be evaluated in vivo. Bioassays have shown the toxicity of plant extracts. Meanwhile, supplementary clinical trial research is necessary to obtain EPIs which are nontoxic at higher concentrations. ${ }^{68}$

\section{Natural Compounds With EPI Property}

Due to the high chemical diversity in plant extracts, it is expected that they are a potential origin of drug resistance modifying substances. The ability of plant extracts to inhibit efflux pumps was discovered by Stavri et al. ${ }^{9}$ In accordance with Table 3 and Figure 4, some plant extracts show EPI properties against $P$. aeruginosa. In addition, the extracts of Thymus maroccanus and Thymus broussonetii represented synergistic properties when mixed with Cam against efflux pump-overexpressing strains of $P$. aeruginosa. ${ }^{75}$ Moreover, ethanolic extracts of Vernonia adoensis and Mangifera indica in the accumulation of rhodamine 6G (R6G) showed the potential properties of EPIs against $P$. aeruginosa. ${ }^{39}$ 
Table 3. Summary of Compounds That Are Active as EPIs Against Pseudomonas aeruginosa

\begin{tabular}{|c|c|c|c|c|}
\hline Compound & Source & Protein & Actions & Ref. \\
\hline \multicolumn{5}{|c|}{ Synthetic Compounds } \\
\hline Peptidomimetic & Synthetic & $\begin{array}{l}\text { MexAB-OprM, MexCD- } \\
\text { Opr], MexEF-OprN }\end{array}$ & $\begin{array}{l}\text { Synergies with Cam, Quinolones, Carb, } \\
\text { Macrolides, and Tet }\end{array}$ & 59 \\
\hline Pyridopyrimidines & Synthetic & MexAB, OprM & $\begin{array}{l}\text { Improves the potency of levofloxacin and } \\
\text { aztreonam }\end{array}$ & 23 \\
\hline Fluoroquinolone analog & Synthetic & MexAB-OprM & $\begin{array}{l}\text { Improves the potency of macrolides and } \\
\text { fluoroquinolones }\end{array}$ & 23 \\
\hline $\begin{array}{l}\text { 4-(3- } \\
\text { morpholinopropylamino)-quinazoline }\end{array}$ & $\begin{array}{l}\text { 4-alkylaminoquinazoline } \\
\text { Derivatives }\end{array}$ & MexAB-OprM & $\begin{array}{l}\text { Reduced MIC of Cam, Nal, } \\
\text { Nor, and Spfx, Increased Cam uptake }\end{array}$ & 64 \\
\hline \multicolumn{5}{|c|}{ Natural Compounds } \\
\hline EA-371 $\alpha$ and EA-371 $\delta$ & $\begin{array}{l}\text { Streptomyces } \\
\text { MF-EA-371-NS1 }\end{array}$ & MexAB-OprM & Reduce MIC of Lev & 65 \\
\hline Geraniol & Helichrysum italicum & - & $\begin{array}{l}\text { Reduced MIC of } \beta \text {-lactams, } \\
\text { Quinolones, and Cam }\end{array}$ & 66 \\
\hline Curcumin & Curcuma longa & - & $\begin{array}{l}\text { Reduced MIC Mem, Carb, Caz, Gen, and } \\
\text { Cip }\end{array}$ & 67 \\
\hline $\begin{array}{l}\text { Lanatoside } \mathrm{C} \text { and } \\
\text { daidzein }\end{array}$ & $\begin{array}{l}\text { Digitalis lanata and Kwao krua, } \\
\text { respectively }\end{array}$ & MexAB-OprM & $\begin{array}{l}\text { Increased uptake of EtBr, Reduced MIC of } \\
\text { Carb and Lev }\end{array}$ & 60 \\
\hline Protocatechuic acid & Camellia sinensis (green tea) & MexAB-OprM & Reduced MIC of Lev & 60 \\
\hline Pheophorbide a & Berberis aetnensis & MexAB-OprM & Synergize with Cip & 68,69 \\
\hline Theobromine & Theobroma cacao & MexAB-OprM & Synergize with Cip & 68,69 \\
\hline \multicolumn{5}{|c|}{ Non-antibacterial drugs } \\
\hline $\begin{array}{l}\text { Amitryptiline, } \\
\text { Trans-chlorprothixene }\end{array}$ & Non-antibiotic drugs & - & Reduced MIC of Pen, Cxm and Tob & 70 \\
\hline Sertraline, chlorpromazine & $\begin{array}{l}\text { Selective Serotonin } \\
\text { Re-uptake Inhibitors }\end{array}$ & MexAB-OprM & Inhibition of Nile Red efflux & 49 \\
\hline
\end{tabular}

Abbreviations: Cam (Chloramphenicol); Carb (Carbanecillin); Caz (Ceftazidime); Cip (Ciprofloxacin); Cxm (Cefuroxime); EtBr (Ethidium Bromide); Gen (Gentamicin); Lev (Levofloxacin); Mem (Meropenem); Nal (Nalidixic acid); Nor (Norfloxacin); Pen (Penicillin); Spfx (Sparfloxacin); Tet (Tetracycline); and Tob (Tobramycin).

Although plant extracts may have no antimicrobial properties singly, when they are applied simultaneously with standard antibiotics, they can act as EPIs and enhance the efficacy of drugs. ${ }^{39,76}$

EPIs derived from microbial origins are somewhat rare. The study of microbial fermentation has led to the identification of 2 new EPIs ${ }^{65}$ which were derived from Streptomyces MFEA-371-NS1, which is a new strain with a close relationship to Streptomyces vellosus. ${ }^{65}$ EA-371 $\alpha^{40}$ and EA-371 $\delta^{41}$ both block the MDR pump of $P$. aeruginosa PAM1032, which overexpresses MexAB-OprM. ${ }^{9}$

To use natural compounds as EPIs in clinical treatments, their spectrum of activity, potency, toxicity, and pharmacokinetics should be determined. Accurate information regarding the binding site of an EPI and the mechanism of inhibition could assist the scientific design of analogs that could overcome these importance issues. ${ }^{57}$

\section{Manners for Studying EPIs}

The most important problem in screening studies for EPIs is that in numerous cases the synergism could be caused by non-specific destruction in the bacterial membrane. As a result, most EPIs, especially PA $\beta \mathrm{N}$, would act similarly against human cells, and would therefore be cytotoxic. Consequently, comprehensive research with the purpose of confirming true EPI action needs to be done. ${ }^{29}$
The rapid expansion of knowledge in molecular biology and biochemistry as well the accessibility of a great amount of bacterial genome data has simplified investigations for understanding the importance of antibiotic transporter inhibitors in drug resistance. To date, diverse biochemical and molecular methods have been employed to reveal EPIs and distinguish their portion in resistance. ${ }^{25}$ To evaluate the phenotypic effectiveness of natural substrates on efflux pumps, both checkerboard and accumulation assays can be applied. These are significant screening tools that allow many EPI potential compounds to be examined easily and quickly. ${ }^{9}$ Another phenotypic method for checking the activity potential of antibiotics by EPIs is the agar doubling dilution method. ${ }^{77}$ As clarified by Lomovskaya et al ${ }^{56}$ for a substance to be certified as an EPI, it should encompass the criteria shown in Table 3. All these criteria can be researched with advanced techniques (Table 4).

\section{Measuring Synergism (MIC of Antibiotics \pm Plant Extracts)} It has been known for a long time that a number of antibiotics show synergism when used simultaneously. The checkerboard microtiter test has been utilized to recognize such agents. This method with variations and modifications has been used to recognize possible inhibitors of efflux pumps. ${ }^{9}$ In this modified method, after MIC determination of the natural substance and antibiotic (A) using standard broth dilution methods, 50 

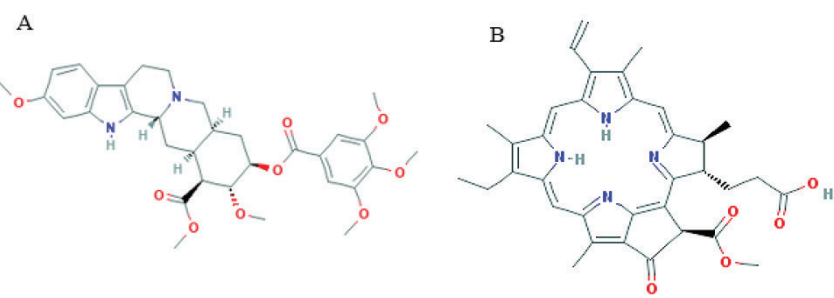

$\mathrm{C}$

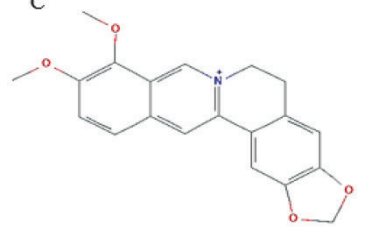

$\mathrm{D}$

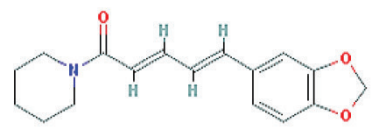

Figure 4. Chemical Structures of 4 Plant Extracts as EPIs, Including Reserpine, Berberine, Piperine, and Pheophorbide.

$\mu \mathrm{L}$ of subinhibitory concentrations for serial dilution for an antibiotic and also $50 \mu \mathrm{L}$ of subinhibitory concentrations of serial dilution for a natural substance (B) (usually $4 \times$ less than the MIC) were combined in a 96-well microtiter plate. ${ }^{79}$ The bacterial strains were adjusted to $10^{6} \mathrm{CFU} / \mathrm{mL}$ cell density and $50 \mu \mathrm{l}$ was used in each well. The fractional inhibitory concentration (FIC) index was determined according to the following formula ${ }^{60}$ :

(MIC of A in mixture/MIC of A singly) + (MIC of B in mixture/MIC of $\mathrm{B}$ singly)

The FIC index was applied to interpret the potency of the combinations. ${ }^{39,60}$ Synergism was considered as FICI $\leq 0.5$, indifferent was considered as a FICI $=0.5-4$, and antagonism was considered as FICI $>4 .{ }^{80}$ The varying amounts of sensitivity of the bacteria to the EPI and drugs may be explained by the varied efflux pump accessibility rates in different isolates and the combination of ingredients present in the extract. ${ }^{81}$

For gram-positive bacteria, one famous inhibitor, e.g., piperine, CCCP, or reserpine, can be used easily as the positive control together with a natural substrate and target antibiotics. $^{9,82}$ Reserpine, a plant alkaloid, was initially extracted from the Rauwolfia vomitoria and was found to block Nor A pumps. ${ }^{9}$ Piperine is also a plant alkaloid extracted from the family Piperaceae such as Piper longum (long pepper) and Piper nigrum (black pepper) and can enhance the accumulation of Cip by Staphylococcus aureus. ${ }^{83}$ According to the, Cip is a substrate of a lot of bacterial efflux pumps, ${ }^{16}$ and primary experiments applied a simple bioassay to recognize plant extracts that synergize with Cip. ${ }^{69}$

On the contrary, because gram-negative bacteria utilize numerous efflux pumps, it is harder to recognize inhibitors that specifically influence one or all of these pumps. Therefore, genotypic methods are preferably utilized to study the role of EPIs in these organisms. ${ }^{84}$ Using phenotypic methods, Touani et al showed that Brassica oleacera var. butyris and Brassica oleacera var. italica extracts containing flavonoids, alkaloids, phenols, sterols, and triterpenes manifested synergistic effects with manifold reductions in the MICs of certain antibiotics examined (antagonistic effects were also detected with some combinations) against MDR $P$. aeruginosa.$^{85}$

In accordance with the report of Garvey et al, all extracts showed higher activity in liquid mediums than in agar. Meanwhile, specific extracts from Melissa officinalis and Levisticum officinale had the greatest activity in connection with antibiotic potentiation with either tetracycline (Te), Cip, or the dye EtBr against gram-negative bacteria. This information proposes that these extracts are inhibitors of efflux. Fractionation of the chloroform extract of L. officinale

Table 4. Required Principles of Compounds to be Certified as EPIs and Their Analyzing Methods

\begin{tabular}{|c|c|c|}
\hline Criteria & Techniques & Reference \\
\hline $\begin{array}{l}\text { It should potentiate the potency of an antibiotic against } \\
\text { generated resistance in result of the overexpression of an } \\
\text { efflux pump. }\end{array}$ & Synergism is measured using checkerboard assay. & 9 \\
\hline $\begin{array}{l}\text { It should enhance the amount of accumulation and reduce } \\
\text { the amount of extrusion for substrates of the efflux pump. }\end{array}$ & $\begin{array}{l}\text { This capability can be measured by fluorescent compound accumulation assays } \\
\text { in the presence and absence of the putative EPI (drug efflux/accumulation assay). }\end{array}$ & 29 \\
\hline $\begin{array}{l}\text { It should not affect the sensitive strains which do not have } \\
\text { the intended efflux pump. }\end{array}$ & $\begin{array}{l}\text { Use of a sensitive strain and a wild type strain (resistant) and then the checkerboard } \\
\text { method can be executed on the resistant strain. }\end{array}$ & 29 \\
\hline $\begin{array}{l}\text { It should not decrease the MIC of antibiotics which are not } \\
\text { extruded. }\end{array}$ & $\begin{array}{l}\text { Use of a sensitive strain and a wild type strain (resistant) and then the checkerboard } \\
\text { method can be executed on the resistant strain. }\end{array}$ & 29 \\
\hline It should not cause penetrable OM. & $\begin{array}{l}\text { Periplasmic } \beta \text {-lactamase property in nitrocefin (a } \beta \text {-lactam) hydrolysis, which alters } \\
\text { from yellow to red when hydrolyzed, can be used. }\end{array}$ & 29 \\
\hline $\begin{array}{l}\text { It should not change the proton gradient available on the } \\
\text { IM; in other words, it should not disturb the membrane } \\
\text { integrity }\end{array}$ & $\begin{array}{l}\text { DNA stains with fluorescence properties such as SYTOX Green or propdium } \\
\text { iodide, which do not pass the intact membrane of bacterial cells, can be used. }\end{array}$ & 78 \\
\hline
\end{tabular}


revealed that of these, falcarindiol had the maximum antibacterial activity and a strong synergistic effect on the activity of Cip. ${ }^{37}$

In a study by Aparna et $\mathrm{al},{ }^{60}$ bioinformatic calculations and checkerboard results proposed that daidzein and lanatoside $C$ are probable inhibitors of the MexAB-OprM efflux system in $P$. aeruginosa. Lanatoside $\mathrm{C}$ in principle is a cardiac glycoside blocker and can increase intracellular $\mathrm{Ca} 2+$ concentration. $^{74}$ The blocking effect of Lanatoside $\mathrm{C}$ on efflux systems could probably be a result of its inhibitory effect on sodium pumps. ${ }^{86}$

Seasotiya et $\mathrm{al}^{81}$ reported that the combination of Cip and Piperine could reduce the amount of MICC against $P$. aeruginosa (MTCC 7453 and MTCC 424) by half, but none of the 35 Indian medicinal herbal extracts could be effective in reducing MIC. Of course, only plants which show no direct antibacterial activity were entered in this study. ${ }^{60}$ This issue proves that the EPI of plant extracts potentially have a limited extent. When was being fractioned a plant extract with the synergism capability, but do not have EPI-like activity none of the its components only, it can be assumed that the efflux inhibitor was either inactivated or lost during fractionation or that it is active in combination with another fraction in the extract from which it was isolated. ${ }^{37}$

\section{Fractionation of Extracts}

When it is realized that an extract can be synergistic with an antibiotic, the extract is fractionated and the effector molecules are revealed. This requires the use of a significant screening method which enables a lot of fractions to be analyzed easily and quickly. Fractionation was performed on silica gel by vacuum liquid chromatography (VLC). Then, the fractions were purified by thin-layer chromatography (TLC) ${ }^{37}$ Eventually, the MIC of antibiotics in the absence and presence of fractions and purified compounds was measured.

\section{Measuring Fluorescent Dye Accumulation \pm Plant Extracts} The general methodology for assessing increases in accumulation is easily done by measuring accumulation in the intracellular concentration marker (fluorescent dyes) in the presence or absence of plant extracts with a spectrophotometer. ${ }^{37,39,60}$ If the natural substance increases the measure of the marker after confronting, it is considered an important efflux inhibitor and vice versa. For example, lanatoside $\mathrm{C}$ and daidzein have shown a notably increased rate in the aggregation of EtBr for $P$. aeruginosa. ${ }^{60,87}$

In an accumulation assay an ionophore (e.g., CCCP or valinomycin) is first used to de-energize bacterial cells. Deenergizing caused no compound to be effluxed until the energize phase. In the next stage, ionophore was eliminated by washing, and then cells were loaded with fluorescent compound. Fluorescent efflux was begun by adding glucose (energize) and EPI. ${ }^{29,56,88}$

The accumulation of fluorescent dyes such as ethidium bromide (EtBr), TMA-DPH, Hoechst 33342 (Bisbenzimide), $N$-phenylnaphthylamine (NPN), and berberine enhances the fluorescence measure inside the cells, but an accumulation of R6G and doxorubicin leads to a reduction in the fluorescence signal. $^{79,89}$
Berberine, isolated from the Berberis genus, is a plant alkaloid and has properties such as EPI against the S. aureus, weak antibacterial activity, and the ability to intercalate with DNA similar to EtBr. ${ }^{39}$ This fluorescent dye makes a powerful yellow when bound to DNA. ${ }^{9}$ Therefore, its accumulation inside cells can be easily monitored by measuring the emitted fluorescence. ${ }^{55}$ Berberine-producing plants synthesize 2 other substances, the flavonolignan 5-MHC-D and the porphyrin Pheophorbide- $\alpha$, which have no antibacterial property, but have EPI activity (Table 3 ). ${ }^{90}$

The main disadvantage of applying fluorescent compounds with this method is that the potential EPI of the compounds could be strongly colored and therefore reduce the accuracy of the measurements..$^{29}$ Recently, Bohnert et al investigated using Nile Red a technique that is dominant on this problem. Nile Red has a high fluorescence yield when it is connected with cell membrane phospholipids; however, it is approximately nonfluorescent in external mediums. ${ }^{91}$

R6G is accumulated mostly in the cytosol by joining to proteins or nucleic acids. Results from an R6G uptake study by Chitemerere et alshowed that Callistemon citrinus extract inhibited antibiotic efflux pumps. Despite the fact that $P$. aeruginosa is a gram-negative bacterium and due to it having a double membrane, it is less sensitive to drugs or extracts. However, Chitemerere et al reported that $P$. aeruginosa after $S$. aureus is the second most sensitive strain to plant essences. ${ }^{39}$

\section{Use of Molecular Methods in Evaluating EPI}

Since approximately all efflux pumps contribute to other systems of resistance in $P$. aeruginosa and a high level of resistance mechanisms hide the influence of the expression of efflux pumps on MICs, obtaining a differential diagnosis using phenotypic antimicrobial methods is difficult. Furthermore, efflux systems can be overexpressed during therapy, which may clarify treatment failures with drugs that are considered useful according to the primary susceptibility profile. ${ }^{23}$ Thus, it is better to use the genotypic test along with phenotypic tests to study the expression of efflux pump genes in P. aeruginosa.

It has been proven that molecular methods are the only ways to survey the expression of efflux systems in clinical strains. The western blotting technique was introduced first, but subsequently, the reverse transcriptase quantitative PCR method (RT-qPCR) quickly became famous because of its greater rapidity and specificity. Thus, RT-qPCRs were expanded to detect and measure the expression of the genes coding for the diverse proteins of an RND pump. These methods stays can be used in clinical laboratories. ${ }^{92}$ One inferential point of the application RT-qPCR by $P$. aeruginosa is that a 2 -fold increment in the overexpression of mexA and mex $\mathrm{B}$ genes leads to an increase in MIC values, while expression of the mexX should be greater ( $\geq 5$-fold) in order to increase antibiotic resistance. ${ }^{93}$

In contrast, reverse transcriptase PCR (RT-PCR) is less costly, but laborious. RT-PCR allows the amplification products to quickly be observed and is easily applicable in clinical laboratories where a real-time PCR device is not available. ${ }^{92}$ Since the quantity of the expressed efflux pumps is not measured, the efficiency of RT-PCR may be lower in 
comparison with real time RT-qPCR. In a study by Jalalvandi et al, the results of RT-PCR revealed that the expression of mexA and mexR genes, related to MexAB-OprM in P. aeruginosa, were significantly reduced after confronting Satureja khuzestanica essence. ${ }^{51}$ Furthermore, there are commercial kits for use in checking the expression of $P$. aeruginosa efflux pumps, such as the mexQ-TesT kit that facilitates the analysis of mexA and mexX genes and the expression of clinical strains versus $\mathrm{PAO} 1$ (wild strain). ${ }^{27}$

\section{Conclusion}

As regards the increasing prevalence of MDR P. aeruginosa, particularly in hospitalized patients, and the restrictions in the utilization of broad spectrum antibiotics in immunocompromised patients, pseudomonas infections are considered to be a developing threat to the community. ${ }^{4}$ There are 3 ways to combat MDR $P$. aeruginosa:

1. Development of novel antibiotics. Although new antibiotics may be helpful in the short term, the organisms rapidly adapt to the changes. ${ }^{94}$

2. Alternative therapy and attention to novel antimicrobial factors, including phages, antibodies, and selective peptides or the use of medicinal natural products. ${ }^{95}$

3. Combination therapy in order to reach bactericidal synergism.

With the lack of progress in novel antimicrobial development, an interest has emerged in the detection of compounds that restore the activity of licensed antibacterial agents that until recently had extraordinary affections against $P$. aeruginosa. ${ }^{37}$ The idea of using a compound which contains a conventional antibiotic and an inhibitor resistance is well verified; co-amoxiclav is a good example. ${ }^{96}$ Another example of a combinational command is the prescription of novel $\beta$-lactamase inhibitors with cephalosporins or penicillins. ${ }^{97}$ However, $\beta$-lactam antibiotics or various classes of aminoglycosides are, unfortunately, ineffective in the treatment of MDR Pseudomonas infections.

Efflux pumps have an important role in developing resistance to antimicrobial agents, particularly in $P$. aeruginosa. For this reason, they can be targets for natural antimicrobial compounds. ${ }^{56}$ Many plant extracts have been recognized as EPIs when applied as adjuvants in combination with the special antibiotics. To fight the MDR P. aeruginosa, a combination of plant extracts with EPI properties and antibiotics would be a better way. ${ }^{51}$

This review has emphasized a number of bacterial EPIs obtained from natural sources, mainly from plants. Some of these substances have remarkable activities and can be optimized in the future. It is suggested that plant extracts must be further studied for their potential to block efflux pumps and these compounds be consumed together with antibiotics as chemotherapeutic agents.

\section{Conflict of Interest Disclosures}

The authors declare they have no conflicts of interest.

\section{References}

1. Hirakata Y, Kondo A, Hoshino K, et al. Efflux pump inhibitors reduce the invasiveness of Pseudomonas aeruginosa. Int J Antimicrob Agents. 2009;34(4):343-346. doi:10.1016/j. ijantimicag.2009.06.007.

2. Alp E, Guven M, Yildiz O, Aygen B, Voss A, Doganay M. Incidence, risk factors and mortality of nosocomial pneumonia in intensive care units: a prospective study. Ann Clin Microbiol Antimicrob. 2004;3:17. doi:10.1186/1476-0711-3-17.

3. Leid JG, Kerr M, Selgado C, et al. Flagellum-mediated biofilm defense mechanisms of Pseudomonas aeruginosa against hostderived lactoferrin. Infect Immun. 2009;77(10):4559-4566. doi:10.1128/iai.00075-09.

4. Nikaido H. Prevention of drug access to bacterial targets: permeability barriers and active efflux. Science. 1994;264(5157):382-388. doi:10.1126/science.8153625.

5. Li XZ, Nikaido H. Efflux-mediated drug resistance in bacteria: an update. Drugs. 2009;69(12):1555-1623. doi:10.2165/11317030000000000-00000.

6. Poole K. Efflux-mediated multiresistance in Gram-negative bacteria. Clin Microbiol Infect. 2004;10(1):12-26. doi:10.1111/ j.1469-0691.2004.00763.x.

7. Poole K, Krebes K, McNally C, Neshat S. Multiple antibiotic resistance in Pseudomonas aeruginosa: evidence for involvement of an efflux operon. J Bacteriol. 1993;175(22):7363-7372. doi:10.1128/jb.175.22.7363-7372.1993.

8. Breidenstein EB, de la Fuente-Nunez C, Hancock RE. Pseudomonas aeruginosa: all roads lead to resistance. Trends Microbiol. 2011;19(8):419-426. doi:10.1016/j.tim.2011.04.005.

9. Stavri M, Piddock LJ, Gibbons S. Bacterial efflux pump inhibitors from natural sources. J Antimicrob Chemother. 2007;59(6):12471260. doi:10.1093/jac/dkl460.

10. Van Bambeke F, Michot JM, Tulkens PM. Antibiotic efflux pumps in eukaryotic cells: occurrence and impact on antibiotic cellular pharmacokinetics, pharmacodynamics and toxicodynamics. J Antimicrob Chemother. 2003;51(5):1067-1077. doi:10.1093/jac/ dkg225.

11. Zechini B, Versace I. Inhibitors of multidrug resistant efflux systems in bacteria. Recent Pat Antiinfect Drug Discov. 2009;4(1):37-50. doi:10.2174/157489109787236256.

12. Chopra I. New developments in tetracycline antibiotics: glycylcyclines and tetracycline efflux pump inhibitors. Drug Resist Updat. 2002;5(3-4):119-125. doi:10.1016/S1368-7646(02)000511.

13. Kumar A, Schweizer HP. Bacterial resistance to antibiotics: active efflux and reduced uptake. Adv Drug Deliv Rev. 2005;57(10):14861513. doi:10.1016/j.addr.2005.04.004.

14. Fernando DM, Kumar A. Resistance-nodulation-division multidrug efflux pumps in gram-negative bacteria: role in virulence. Antibiotics (Basel). 2013;2(1):163-181. doi:10.3390/ antibiotics2010163.

15. Vidal-Aroca F, Meng A, Minz T, Page MG, Dreier J. Use of resazurin to detect mefloquine as an efflux-pump inhibitor in Pseudomonas aeruginosa and Escherichia coli. J Microbiol Methods. 2009;79(2):232-237. doi:10.1016/j.mimet.2009.09.021.

16. Piddock LJ. Clinically relevant chromosomally encoded multidrug resistance efflux pumps in bacteria. Clin Microbiol Rev. 2006;19(2):382-402. doi:10.1128/cmr.19.2.382-402.2006.

17. Van Bambeke F, Balzi E, Tulkens PM. Antibiotic efflux pumps. Biochem Pharmacol. 2000;60(4):457-470.

18. Zgurskaya HI, Nikaido H. AcrA is a highly asymmetric protein capable of spanning the periplasm. J Mol Biol. 1999;285(1):409420. doi:10.1006/jmbi.1998.2313.

19. Tegos GP, Haynes M, Strouse JJ, et al. Microbial efflux pump inhibition: tactics and strategies. Curr Pharm Des. 2011;17(13):1291-1302.

20. Misra R, Bavro VN. Assembly and transport mechanism of tripartite drug efflux systems. Biochim Biophys Acta. 2009;1794(5):817825. doi:10.1016/j.bbapap.2009.02.017.

21. Han XY, De I, Jacobson KL. Rapidly growing mycobacteria: clinical and microbiologic studies of 115 cases. Am J Clin Pathol. 
2007;128(4):612-621. doi:10.1309/1 KB2GKYT1BUEYLB5.

22. Masuda N, Sakagawa E, Ohya S, Gotoh N, Tsujimoto H, Nishino T. Substrate specificities of MexAB-OprM, MexCD-OprJ, and MexXYoprM efflux pumps in Pseudomonas aeruginosa. Antimicrob Agents Chemother. 2000;44(12):3322-3327. doi:10.1128/ AAC.44.12.3322-3327.2000.

23. Van Bambeke F, Pages JM, Lee VJ. Inhibitors of bacterial efflux pumps as adjuvants in antibiotic treatments and diagnostic tools for detection of resistance by efflux. Recent Pat Antiinfect Drug Discov. 2006;1(2):157-175. doi:10.2174/157489106777452692.

24. Strateva T, Yordanov D. Pseudomonas aeruginosa - a phenomenon of bacterial resistance. J Med Microbiol. 2009;58(Pt 9):11331148. doi:10.1099/jmm.0.009142-0.

25. Li XZ, Plesiat P, Nikaido $\mathrm{H}$. The challenge of efflux-mediated antibiotic resistance in gram-negative bacteria. Clin Microbiol Rev. 2015;28(2):337-418. doi:10.1128/cmr.00117-14.

26. Zhanel GG, Hoban DJ, Schurek K, Karlowsky JA. Role of efflux mechanisms on fluoroquinolone resistance in Streptococcus pneumoniae and Pseudomonas aeruginosa. Int J Antimicrob Agents. 2004;24(6):529-535. doi:10.1016/j.ijantimicag.2004.08.003.

27. Avrain L, Mertens P, Van Bambeke F. RND efflux pumps in P. aeruginosa: an underestimated resistance mechanism. 2013; 2628.

28. Lister PD, Wolter DJ, Hanson ND. Antibacterial-resistant Pseudomonas aeruginosa: clinical impact and complex regulation of chromosomally encoded resistance mechanisms. Clin Microbiol Rev. 2009;22(4):582-610. doi:10.1128/cmr.00040-09.

29. Venter H, Mowla R, Ohene-Agyei T, Ma S. RND-type drug e ffl ux pumps from Gram-negative bacteria: molecular mechanism and inhibition. Front Microbiol. 2015;6:377. doi:10.3389/ fmicb.2015.00377.

30. Welch A, Awah CU, Jing S, van Veen HW, Venter H. Promiscuous partnering and independent activity of MexB, the multidrug transporter protein from Pseudomonas aeruginosa. Biochem J. 2010;430(2):355-364. doi:10.1042/bj20091860.

31. Poole K, Srikumar R. Multidrug efflux in Pseudomonas aeruginosa: components, mechanisms and clinical significance. Curr Top Med Chem. 2001;1(1):59-71. doi:10.2174/1568026013395605.

32. Poole K. Efflux-mediated antimicrobial resistance. J Antimicrob Chemother. 2005;56(1):20-51. doi:10.1093/jac/dki171.

33. Jeannot K, Elsen S, Kohler T, Attree I, van Delden C, Plesiat P. Resistance and virulence of Pseudomonas aeruginosa clinical strains overproducing the MexCD-OprJ efflux pump. Antimicrob Agents Chemother. 2008;52(7):2455-2462. doi:10.1128/ aac.01107-07.

34. Dean CR, Visalli MA, Projan SJ, Sum PE, Bradford PA. Effluxmediated resistance to tigecycline (GAR-936) in Pseudomonas aeruginosa PAO1. Antimicrob Agents Chemother. 2003;47(3):972978. doi:10.1128/AAC.47.3.972-978.2003.

35. He GX, Kuroda T, Mima T, Morita Y, Mizushima T, Tsuchiya T. An $\mathrm{H}(+)$-coupled multidrug efflux pump, PmpM, a member of the MATE family of transporters, from Pseudomonas aeruginosa. J Bacteriol. 2004;186(1):262-265.

36. Gales AC, Jones RN, Sader HS. Global assessment of the antimicrobial activity of polymyxin B against 54731 clinical isolates of gram-negative bacilli: report from the SENTRY antimicrobial surveillance programme (2001-2004). Clin Microbiol Infect. 2006;12(4):315-321. doi:10.1111/j.1469-0691.2005.01351.x.

37. Garvey MI, Rahman MM, Gibbons S, Piddock LJ. Medicinal plant extracts with efflux inhibitory activity against gramnegative bacteria. Int J Antimicrob Agents. 2011;37(2):145-151. doi:10.1016/j.ijantimicag.2010.10.027.

38. Pages JM, Amaral L. Mechanisms of drug efflux and strategies to combat them: challenging the efflux pump of Gram-negative bacteria. Biochim Biophys Acta. 2009;1794(5):826-833. doi:10.1016/j.bbapap.2008.12.011.

39. Chitemerere TA, Mukanganyama S. In vitro antibacterial activity of selected medicinal plants from Zimbabwe. Afr J Plant Sci
Biotechnol. 2011;5(1):1-7.

40. Mahamoud A, Chevalier J, Alibert-Franco S, Kern WV, Pages JM. Antibiotic efflux pumps in gram-negative bacteria: the inhibitor response strategy. J Antimicrob Chemother. 2007;59(6):12231229. doi:10.1093/jac/dkl493.

41. Ambudkar SV, Dey S, Hrycyna CA, Ramachandra M, Pastan I, Gottesman MM. Biochemical, cellular, and pharmacological aspects of the multidrug transporter. Annu Rev Pharmacol Toxicol. 1999;39:361-398. doi:10.1146/annurev.pharmtox.39.1.361.

42. Eid SY, El-Readi MZ, Fatani SH, Eldin EE, Wink M. Natural products modulate the multifactorial multidrug resistance of cancer. Pharmacol Pharm. 2015;6(3):146-176. doi:10.4236/ pp.2015.63017.

43. HohlAF, Frei R, PunterV, etal. International multicenter investigation of LB20304, a new fluoronaphthyridone. Clin Microbiol Infect. 1998;4(5):280-284. doi:10.1111/j.1469-0691.1998.tb00057.x.

44. Vadlapatla RK, Vadlapudi AD, Kwatra D, Pal D, Mitra AK. Differential effect of P-gp and MRP2 on cellular translocation of gemifloxacin. Int J Pharm. 2011;420(1):26-33. doi:10.1016/j. ijpharm.2011.08.009.

45. Kullak-Ublick GA, Becker MB. Regulation of drug and bile salt transporters in liver and intestine. Drug Metab Rev. 2003;35(4):305317. doi:10.1081/DMR-120026398.

46. Rice A, Liu Y, Michaelis ML, Himes RH, Georg Gl, Audus KL. Chemical modification of paclitaxel (Taxol) reduces P-glycoprotein interactions and increases permeation across the blood-brain barrier in vitro and in situ. J Med Chem. 2005;48(3):832-838. doi:10.1021/jm040114b.

47. Colegate SM, Molyneux RJ, editors. Bioactive natural products: detection, isolation, and structural determination. 2nd ed. Boca Raton: CRC Press; 2008.

48. Conseil G, Baubichon-Cortay H, Dayan G, Jault JM, Barron D, Di Pietro A. Flavonoids: a class of modulators with bifunctional interactions at vicinal ATP- and steroid-binding sites on mouse P-glycoprotein. Proc Natl Acad Sci U S A. 1998;95(17):98319836. doi:10.1073/pnas.95.17.9831.

49. Bohnert JA, Szymaniak-Vits M, Schuster S, Kern WV. Efflux inhibition by selective serotonin reuptake inhibitors in Escherichia coli. J Antimicrob Chemother. 2011;66(9):2057-2060. doi:10.1093/jac/dkr258.

50. Ricci V, Busby SJ, Piddock LJ. Regulation of RamA by RamR in Salmonella enterica serovar Typhimurium: isolation of a RamR superrepressor. Antimicrob Agents Chemother. 2012;56(11):60376040. doi:10.1128/aac.01320-12.

51. Jalalvandi N, Bahador A, Zahedi B, Saghi H, Esmaeili D. The study of inhibitory effects of Satureja khuzestanica essence against mexa and mexr efflux genes of Pseudomonas aeruginosa by Rt-Pcr. Int J Biotechnol. 2015;4(1):1-8. doi:10.18488/ journal.57/2015.4.1/57.1.1.8.

52. Tikhonova EB, Yamada Y, Zgurskaya HI. Sequential mechanism of assembly of multidrug efflux pump AcrAB-TolC. Chem Biol. 2011;18(4):454-463. doi:10.1016/j.chembiol.2011.02.011.

53. Venter H, Shilling RA, Velamakanni S, Balakrishnan L, Van Veen HW. An ABC transporter with a secondary-active multidrug translocator domain. Nature. 2003;426(6968):866-870. doi:10.1038/nature02173.

54. Askoura M, Mottawea W, Abujamel T, Taher I. Efflux pump inhibitors (EPIs) as new antimicrobial agents against Pseudomonas aeruginosa. Libyan J Med. 2011;6. doi:10.3402/ljm.v6i0.5870.

55. Tegos G, Stermitz FR, Lomovskaya O, Lewis K. Multidrug pump inhibitors uncover remarkable activity of plant antimicrobials. Antimicrob Agents Chemother. 2002;46(10):3133-3141. doi:10.1128/AAC.46.10.3133-3141.2002.

56. Lomovskaya O, Warren MS, Lee A, et al. Identification and characterization of inhibitors of multidrug resistance efflux pumps in Pseudomonas aeruginosa: novel agents for combination therapy. Antimicrob Agents Chemother. 2001;45(1):105-116. doi:10.1128/ aac.45.1.105-116.2001. 
57. Opperman TJ, Nguyen ST. Recent advances toward a molecular mechanism of efflux pump inhibition. Front Microbiol. 2015;6:421. doi:10.3389/fmicb.2015.00421.

58. Okandeji BO, Greenwald DM, Wroten J, Sello JK. Synthesis and evaluation of inhibitors of bacterial drug efflux pumps of the major facilitator superfamily. Bioorg Med Chem. 2011;19(24):76797689. doi:10.1016/j.bmc.2011.10.011.

59. Lomovskaya O, Bostian KA. Practical applications and feasibility of efflux pump inhibitors in the clinic--a vision for applied use. Biochem Pharmacol. 2006;71(7):910-918. doi:10.1016/j. bcp.2005.12.008.

60. Aparna V, Dineshkumar K, Mohanalakshmi N, Velmurugan D, Hopper W. Identification of natural compound inhibitors for multidrug efflux pumps of Escherichia coli and Pseudomonas aeruginosa using in silico high-throughput virtual screening and in vitro validation. PLoS One. 2014;9(7):e101840. doi:10.1371/ journal.pone.0101840.

61. Bhardwaj AK, Mohanty P. Bacterial efflux pumps involved in multidrug resistance and their inhibitors: rejuvinating the antimicrobial chemotherapy. Recent Pat Antiinfect Drug Discov. 2012;7(1):73-89. doi:10.2174/157489112799829710.

62. Mahamoud A, Chevalier J, Davin-Regli A, Barbe J, Pages JM. Quinoline derivatives as promising inhibitors of antibiotic efflux pump in multidrug resistant Enterobacter aerogenes isolates. Curr Drug Targets. 2006;7(7):843-847. doi:10.2174/138945006777709557.

63. Wang D, Gao F. Quinazoline derivatives: synthesis and bioactivities. Chem Cent J. 2013;7(1):95. doi:10.1186/1752153x-7-95.

64. Mahamoud A, Chevalier J, Baitiche M, Adam E, Pages JM. An alkylaminoquinazoline restores antibiotic activity in gramnegative resistant isolates. Microbiology. 2011;157(Pt 2):566-571. doi:10.1099/mic.0.045716-0.

65. Lee MD, GalazzoJL, Staley AL, etal. Microbial fermentation-derived inhibitors of efflux-pump-mediated drug resistance. Farmaco. 2001;56(1-2):81-85. doi:10.1016/S0014-827X(01)01002-3.

66. Lorenzi V, Muselli A, Bernardini AF, et al. Geraniol restores antibiotic activities against multidrug-resistant isolates from gramnegative species. Antimicrob Agents Chemother. 2009;53(5):22092211. doi:10.1128/aac.00919-08.

67. Negi N, Prakash P, Gupta ML, Mohapatra TM. Possible role of curcumin as an efflux pump inhibitor in multi drug resistant clinical isolates of Pseudomonas aeruginosa. J Clin Diagn Res. 2014;8(10):Dc04-07. doi:10.7860/jcdr/2014/8329.4965.

68. Rana T, Singh S, Kaur K, Pathania K, Farooq U. A review on efflux pump inhibitors of medically important bacteria from plant sources. Int J Pharm Sci Rev Res. 2014;26(2):101-111.

69. Piddock LJ, Garvey MI, Rahman MM, Gibbons S. Natural and synthetic compounds such as trimethoprim behave as inhibitors of efflux in gram-negative bacteria. J Antimicrob Chemother. 2010;65(6):1215-1223. doi:10.1093/jac/dkq079.

70. Kristiansen JE, Thomsen VF, Martins A, Viveiros M, Amaral L. Nonantibiotics reverse resistance of bacteria to antibiotics. In Vivo. 2010;24(5):751-754

71. Amusan OOG, Sukati NA, Dlamini PS, Sibandze FG. Some Swazi phytomedicines and their constituents. Afr J Biotechnol. 2007;6(3):267-272.

72. Ruegg $\mathrm{T}$, Calderon Al, Queiroz EF, et al. 3-Farnesyl-2hydroxybenzoic acid is a new anti-Helicobacter pylori compound from Piper multiplinervium. J Ethnopharmacol. 2006;103(3):461467. doi:10.1016/j.jep.2005.09.014.

73. Cherigo L, Pereda-Miranda R, Fragoso-Serrano M, Jacobo-Herrera N, Kaatz GW, Gibbons S. Inhibitors of bacterial multidrug efflux pumps from the resin glycosides of Ipomoea murucoides. J Nat Prod. 2008;71(6):1037-1045. doi:10.1021/np800148w.

74. Kumar A, Khan IA, Koul S, et al. Novel structural analogues of piperine as inhibitors of the NorA efflux pump of Staphylococcus aureus. J Antimicrob Chemother. 2008;61(6):1270-1276. doi:10.1093/jac/dkn088.

75. Fadli M, Chevalier J, Saad A, Mezrioui NE, Hassani L, Pages JM. Essential oils from Moroccan plants as potential chemosensitisers restoring antibiotic activity in resistant gram-negative bacteria. Int J Antimicrob Agents. 2011;38(4):325-330. doi:10.1016/j. ijantimicag.2011.05.005.

76. Kamatou GPP, van Zyl RL, van Vuuren SF, et al. Chemical composition, leaf trichome types and biological activities of the essential oils of four related Salvia species indigenous to southern Africa. J Essent Oil Res. 2006;18:72-79.

77. Andrews JM. Determination of minimum inhibitory concentrations. J Antimicrob Chemother. 2001;48 Suppl 1:5-16. doi:10.1093/ jac/48.suppl_1.5.

78. Nakashima R, Sakurai K, Yamasaki S, Nishino K, Yamaguchi A. Structures of the multidrug exporter AcrB reveal a proximal multisite drug-binding pocket. Nature. 2011;480(7378):565-569. doi:10.1038/nature10641.

79. Ohene-Agyei T, Mowla R, Rahman T, Venter H. Phytochemicals increase the antibacterial activity of antibiotics by acting on a drug efflux pump. Microbiologyopen. 2014;3(6):885-896. doi:10.1002/ mbo3.212.

80. Odds FC. Synergy, antagonism, and what the chequerboard puts between them. J Antimicrob Chemother. 2003;52(1):1. doi:10.1093/jac/dkg301.

81. Seasotiya L, Dalal S. Screening of Indian medicinal plants as efflux pump inhibitors of fluoroquinolones. J Pharmacogn Phytochem. 2014;3(1):235-241.

82. Neyfakh AA, Bidnenko VE, Chen LB. Efflux-mediated multidrug resistance in Bacillus subtilis: similarities and dissimilarities with the mammalian system. Proc Natl Acad Sci U S A. 1991;88(11):47814785.

83. Khan IA, Mirza ZM, Kumar A, Verma V, Qazi GN. Piperine, a phytochemical potentiator of ciprofloxacin against Staphylococcus aureus. Antimicrob Agents Chemother. 2006;50(2):810-812. doi:10.1128/aac.50.2.810-812.2006.

84. Zhanel GG, Hoban DJ, Schurek K, Karlowsky JA. Role of efflux mechanisms on fluoroquinolone resistance in Streptococcus pneumoniae and Pseudomonas aeruginosa. Int J Antimicrob Agents. 2004;24(6):529-535. doi:10.1016/j.ijantimicag.2004.08.003.

85. Touani FK, Seukep AJ, Djeussi DE, Fankam AG, Noumedem JA, Kuete V. Antibiotic-potentiation activities of four Cameroonian dietary plants against multidrug-resistant gram-negative bacteria expressing efflux pumps. BMC Complement Altern Med. 2014;14:258. doi:10.1186/1472-6882-14-258.

86. Melero C, Medarde M, San Feliciano A. A short review on cardiotonic steroids and their aminoguanidine analogues. Molecules. 2000;5(1):51-81. doi:10.3390/50100051.

87. Ghisalberti D, Mahamoud A, Chevalier J, et al. Chloroquinolines block antibiotic efflux pumps in antibiotic-resistant Enterobacter aerogenes isolates. Int J Antimicrob Agents. 2006;27(6):565-569. doi:10.1016/j.ijantimicag.2006.03.010.

88. Seeger MA, von Ballmoos C, Eicher T, et al. Engineered disulfide bonds support the functional rotation mechanism of multidrug efflux pump AcrB. Nat Struct Mol Biol. 2008;15(2):199-205. doi:10.1038/nsmb.1379.

89. Cha HJ, Muller RT, Pos KM. Switch-loop flexibility affects transport of large drugs by the promiscuous AcrB multidrug efflux transporter. Antimicrob Agents Chemother. 2014;58(8):47674772. doi:10.1128/aac.02733-13.

90. Musumeci R, Speciale A, Costanzo R, et al. Berberis aetnensis C. Presl. extracts: antimicrobial properties and interaction with ciprofloxacin. Int J Antimicrob Agents. 2003;22(1):48-53.

91. Bohnert JA, Karamian B, Nikaido H. Optimized Nile Red efflux assay of AcrAB-TolC multidrug efflux system shows competition between substrates. Antimicrob Agents Chemother. 2010;54(9):3770-3775. doi:10.1128/aac.00620-10.

92. Poonsuk K, Chuanchuen R. Contribution of the MexXY multidrug efflux pump and other chromosomal mechanisms on 
aminoglycoside resistance in Pseudomonas aeruginosa isolates from canine and feline infections. JVet Med Sci. 2012;74(12):15751582. doi:10.1292/jvms.12-0239.

93. Avrain L, Hocquet D, Laurent T, Leclipteux T, Mertens P. Pre-RealTime PCR steps standardization for appropriate interpretation of mexA and mexX gene expression by mex Q-Test in P. aeruginosa. ECCMID; 2010:590.

94. Boucher HW, Talbot GH, Bradley JS, et al. Bad bugs, no drugs: no ESKAPE! An update from the Infectious Diseases Society of America. Clin Infect Dis. 2009;48(1):1-12. doi:10.1086/595011.

95. Raja A, Gajalakshmi P, Mohamed Mahroop Raja M. Drugs from the natural bio sources for human disease. Int J Pharmacol. 2010;6(4):360-363. doi:10.3923/ijp.2010.360.363.

96. Stermitz FR, Lorenz P, Tawara JN, Zenewicz LA, Lewis K. Synergy in a medicinal plant: antimicrobial action of berberine potentiated by $5^{\prime}$-methoxyhydnocarpin, a multidrug pump inhibitor. Proc Natl Acad Sci U S A. 2000;97(4):1433-1437. doi:10.1073/ pnas.030540597.

97. Page MG, Heim J. Prospects for the next anti-Pseudomonas drug. Curr Opin Pharmacol. 2009;9(5):558-565. doi:10.1016/j. coph.2009.08.006. 